\title{
Causes of anorexia in untreated hyperthyroidism: a prospective study
}

\author{
Wei-Xin Dai, Xun-Wu Meng
}

\begin{abstract}
Seventeen consecutive patients (mean (SD) 46 (11) years) with untreated hyperthyroidism and anorexia and 29 patients (35 (9) years) with untreated hyperthyroidism without anorexia were studied. The study was conducted at the thyroid clinic of the PUMC Hospital, Beijing, China from March to August 1997. The patients' ages, serum free calcium, liver function and emotional state, specifically the level of anxiety (using the self anxiety scale, Chinese version), were compared before and/or after antithyroid drug treatment in the two groups. This prospective study suggested that the causes of anorexia in untreated hyperthyroidism are complicated. Older age, abnormal liver function, and the level of anxiety are significantly related to anorexia in untreated hyperthyroidism, but hypercalcaemia was not confirmed to be related to anorexia in the study.

(Postgrad Med f 2000;76:292-294)
\end{abstract}

Keywords: hypercalcaemia; hyperthyroidism; anorexia; anxiety

Patients with hyperthyroidism present with increased adrenal activity and a high metabolic rate. Their gastroenterological symptoms include hunger, polyphagia, and diarrhoea. Some patients complain of anorexia, nausea, and even vomiting. The cause of anorexia in hyperthyroidism is unknown. Some authors have reported that hyperthyroidism with hypercalcaemia could appear as anorexia, nausea, and possibly vomiting and after correcting the hypercalcaemia the symptoms of anorexia, nausea, and vomiting disappear immediately. These descriptions, though, are case reports. ${ }^{1-4}$

We conducted a prospective study of risk factors for anorexia and the relationship between hypercalcaemia and anorexia in hyperthyroidism.

\section{Patients and methods}

We studied 17 consecutive patients with untreated hyperthyroidism with anorexia (group A) and 29 consecutive patients with untreated hyperthyroidism without anorexia (group B) in the thyroid clinic of the PUMC hospital from March to August 1997. All of patients presented with weight loss, heat intolerance, and palpitations. Liver function (alanine aminotransferase (ALT) and aspartate aminotransferase (AST)), serum free calcium, total calcium, and thyroid hormones were measured.
Before the treatment, the level of anxiety was measured by the Chinese version of the self anxiety scale, which is a 20 item, self evaluating, validated questionnaire for anxiety measurement with a total score of 80 . Patients scored items from 1 to 4 ; the more they scored, the higher the level of anxiety they were experiencing.

The hyperthyroidism was treated with an antithyroid drug (methimazole $10 \mathrm{mg}$ or propylthiouracil $100 \mathrm{mg}$ three times a day) for two to three months. In addition, group A was treated with supportive therapy, including infusions of glucose, water, potassium, vitamins, and sedation. After two to three months of antithyroid drug therapy, the hyperthyroidism in all the patients was controlled, their thyroid hormones fell to the normal range, and their serum free calcium, liver function (ALT and AST) and thyroid hormones were measured again.

\section{Results}

Group A included 13 females and four males, their mean (SD) age was 46 (11) years (range 23-60); group B included 20 females and nine males, their age was 35 (9) years (range 18-60). The difference in sex ratio between two groups was not significant $\left(\chi^{2}\right.$ test, $\mathrm{p}>0.05)$; the age of group A was significantly older than of group $\mathrm{B}(t$ test, $\mathrm{p}<0.01)$.

The serum free calcium and liver function between two groups before antithyroid drug treatment were compared. Before antithyroid drug treatment, the serum free calcium of groups A and B was, respectively, 1.28 (0.08) $\mathrm{mmol} / \mathrm{l}$ and $1.27(0.08) \mathrm{mmol} / \mathrm{l}$. The serum free calcium in group A and B was significantly higher than a normal control group of healthy volunteers $(1.18(0.05) \mathrm{mmol} / \mathrm{l}, \mathrm{n}=86, t$ test, $\mathrm{p}<0.01$ ), but there was no significant difference in serum free calcium between groups $\mathrm{A}$ and $\mathrm{B}$ ( $t$ test, $\mathrm{p}>0.05)$. If we define serum free calcium above $1.28 \mathrm{mmol} / 1$ ( $\geqslant$ normal mean + 2SD) as hypercalcaemia, five cases in groups $\mathrm{A}$ and nine cases in group B had hypercalcaemia; there was no difference between two groups $\left(\chi^{2}=0.0176, \mathrm{p}>0.05\right)$. Groups $\mathrm{A}$ and $\mathrm{B}$ had eight and five cases with abnormal liver function (raised ALT and/or raised AST) respectively; there were more patients with abnormal liver function in group $\mathrm{A}$ than in group $\mathrm{B}\left(\chi^{2}\right.$ test, $\mathrm{p}<0.01)$.

When the anxiety score was compared between the two groups the mean (SD) score in group A was 64 (10) and in group B 51 (7); this was significantly higher in group $A$ than in group B ( $t$ test, $\mathrm{p}<0.05)$. We divided all 46 cases into two further groups by age: group C $(n=19)$, older than 40 , had an anxiety score of 
Table 1 Comparison serum free calcium, hypercalcaemia, abnormal liver function, and anxiety score before the antithyroid drug treatment

\begin{tabular}{llllll}
\hline & $\begin{array}{l}\text { Age } \\
\text { (years) }\end{array}$ & $\begin{array}{l}\text { Mean (SD) serum free } \\
\text { calcium (mmol/l) }\end{array}$ & $\begin{array}{l}\text { Hypercalcaemia } \\
\text { (cases) }\end{array}$ & $\begin{array}{l}\text { Abnormal liver } \\
\text { function (cases) }\end{array}$ & $\begin{array}{l}\text { Mean (SD) } \\
\text { anxiety score }\end{array}$ \\
\hline Group A (n=17) & $46(11)$ & $1.28(0.08)$ & 5 & 8 & $64(10)$ \\
Group B (n=29) & $35(9)$ & $1.27(0.08)$ & 9 & 5 & $51(7)$ \\
p Value & $<0.01$ & $>0.05$ & $>0.05$ & $<0.01$ & $<0.05$ \\
\hline
\end{tabular}

64 (9) and group D ( $\mathrm{n}=27)$, less or equal to 40, had a score of 55 (10). There was no significant difference between groups C and D ( $t$ test, $\mathrm{p}>0.05$ )

The serum free calcium and liver function between two groups was compared after treatment. After two to three months of antithyroid drug treatment, hyperthyroidism in all the cases was controlled. Thyroid hormones in groups $\mathrm{A}$ and $\mathrm{B}$ returned to the normal range. The mean (SD) serum free calcium in groups $\mathrm{A}$ and $\mathrm{B}$ was respectively $1.18(0.05)$ and 1.17 (0.06) $\mathrm{mmol} / \mathrm{l}$. There was no difference in serum free calcium between controls and groups $\mathrm{A}$ or $\mathrm{B}$ ( $t$ test, $\mathrm{p}>0.05)$ The liver function in all patients was normal.

See table 1 for the results before treatment.

\section{Discussion}

Thyroid hormones stimulate bone turnover and promote calcium release from bone to circulation and cause an increase in serum calcium. Hyperthyroidism is thus one cause of hypercalcaemia, ${ }^{56}$ and the rate of hypercalcaemia in hyperthyroidism ranges from $15 \%$ to $30 \%{ }^{67}$

Excess thyroid hormones in the blood can lead to a higher metabolism and more activity in the gastrointestinal system. Patients with hyperthyroidism often complain of polyphagia, hunger, and diarrhoea. Some patients with hyperthyroidism complain of anorexia, nausea, and even vomiting. The cause of anorexia in hyperthyroidism is unknown. Some authors reported that patients with hyperthyroidism and anorexia, nausea, and vomiting had high serum calcium concentrations, ${ }^{1-4}$ and after the hypercalcaemia was corrected, these symptoms disappeared immediately. These authors believe that hypercalcaemia can cause anorexia in hyperthyroidism, but these papers were case reports, not prospective investigations.

The purpose of our study was to investigate the relationship between anorexia and hypercalcaemia, and the risk factors of anorexia in hyperthyroidism. We did a prospective study of patients with hyperthyroidism with and without anorexia who presented to our thyroid clinic. Our results suggest that although the serum free calcium of the group with anorexia was significantly higher than normal range, that of the group without anorexia was also significantly higher. There was no difference in serum free calcium between the two groups. There was also no difference in the presence of hypercalcaemia in the two groups.

Our results suggest that hyercalcaemia can be present in patients with hyperthyroidism with and without anorexia. In contrast, patients with hyperthyroidism with anorexia can have a normal calcium concentration. We cannot confirm hypercalcaemia as the cause of anorexia in our study.

We also found that the group with anorexia was older than the group without. In the clinic, young patients with hyperthyroidism often present with polyphagia, hunger, but increased appetite is rare in older patient with hyperthyroidism. Old age may be a cause of anorexia in hyperthyroidism; this is similar to that of older patients with apathetic hyperthyroidism.

We measured liver function in the two groups and the rate of abnormal liver function in the group with anorexia was significantly higher than in the group without $(p<0.01)$, so we conclude that the abnormal liver function was related to the anorexia in hyperthyroidism. Gurlek et al found $60.5 \%$ patients with hyperthyroidism had at least one abnormal liver function at diagnosis. ${ }^{8}$

We noticed that some patients with hyperthyroidism presented with anxiety in our clinic. In our study, we have found that patients with anorexia had a higher anxiety score than those without before the treatment. In order to avoid the effect of age on anxiety, we divided all 46 cases into two groups, older or younger than 40, and there was no significant difference for anxiety score between two groups. We think the anxiety may be a risk factor of anorexia in hyperthyroidism. Fonseca et $a l^{9}$ and Rolla et $a l^{10}$ reported that hyperthyroidism presented with anorexia and anxiety disorder, but the serum calcium was normal. ${ }^{10}$ We encountered a patient in our emergency department who presented with anorexia, repeated nausea and vomiting and severe anxiety; his medical history included hyperthyroidism. His serum free calcium was normal $(1.26 \mathrm{mmol} / \mathrm{l})$, thyroid hormones were completely normal, and after sedation and supportive therapy, the patient's nausea and vomiting disappeared immediately.

After two to three months of antithyroid drug therapy, the hyperthyroidism of all the patients was controlled. Thyroid hormones were normal and the serum free calcium concentration and liver function had returned completely to normal. The symptoms of anorexia and hyperthyroidism disappeared.

We conclude that our prospective study cannot confirm hypercalcaemia as the cause of anorexia in hyperthyroidism. Old age, abnormal liver function, and anxiety were the risk factors for anorexia in hyperthyroidism.

1 Twycross RG, Marks V. Symptomatic hypercalcaemia in thyrotoxicosis. BMF 1970;ii:701-3.

2 Tan TT, Alzaid AA, Sutcliffe N, et al. Treatment of hypercalcaemia in thyrotoxicosis with aminohydroxypropylidene diphosphanate. Postgrad Med f 1988;64: propyliden. 
3 Hedman I, Tisell LE. Life-threatening hypercalcemia in a case of thyrotoxicosis: clinical features and management. Acta Chir Scand 1985;151:487-9.

4 Ghose RR, Palmer DJ, Yaqoob $M$, et al. Abdominal symptoms, hypercalcaemia and apathetic hyperthyroidism: treatment with pamidronate. Br f Clin Pract 1994;48: 163-4.

5 Rude RK, Oldham SB, Singer FR, et al. Treatment of thyrotoxic hypercalcemia with propranolol. $N$ Engl $f \mathrm{Med}$ 1976;294:431-3.

6 Baxter JD, Bondy PK. Hypercalcemia of thyrotoxicosis. Ann Intern Med 1966;65:429-42.
7 Dai WX, Meng XW, Bai Y, et al. Abnormal metabolism of serum free calcium, calcium and phosphate of serum and urine on Graves' disease before and after antithyroid therapy. Chin 7 Endocrinol Metab 1993;9:83-5.

8 Gurlek A, Cobankara V, Bayraktar M. Liver tests in hyperthyroidism: effect of antithyroid therapy. F Clin Gastroenterol 1997;24:180-3.

9 Fonseca V, Wakeling A, Havad CW. Hyperthyroidism and eating disorders. BMF 1990;301:322-33.

10 Rolla AR, El-Haji GA, Goldstein HH. Untreated thyrotoxicosis as a manifestation of anorexia nervosa. $\mathrm{Am} \mathcal{f} \mathrm{Med}$ 1986;81:163-5. 